\title{
Symptoms of depression and anxiety in Indonesian medical students: association with coping strategy and resilience
}

\author{
Adhitya Sigit Ramadianto*, Irmia Kusumadewi, Feranindhya Agiananda and Natalia Widiasih Raharjanti
}

\begin{abstract}
Background: Depression and anxiety are prevalent mental health issues among medical students due to the various challenges during medical education. These issues affect not only their quality of life, but also their academic and professional development. Coping strategy and resilience are two factors that may influence students' mental health outcomes. Data of medical student mental health in Indonesia is scarce, hampering efforts to systematically address the problem. Hence, this study aims to estimate the prevalence of depressive and anxiety symptoms in Indonesian medical students, and their association with coping strategy and resilience.

Methods: Undergraduate medical students from each year of study (Preclinical Year 1 to 4, Clinical Year 1 and 2) in the Faculty of Medicine Universitas Indonesia were randomly selected to participate in this cross-sectional study. The study questionnaire included sociodemographic characteristics, Depression Anxiety Stress Scale (DASS) to measure symptoms of depression and anxiety, Brief COPE to measure coping strategy, and Connor-Davidson Resilience Scale (CD-RISC) to measure resilience. Scores of depression and anxiety symptoms were analyzed by comparing them between different sociodemographic groups and by measuring their correlation with coping strategies and resilience. Multiple regression analyses were conducted to identify predictors of depression and anxiety symptoms.

Results: Among 532 respondents, 22.2\% reported symptoms of depression and 48,1\% reported anxiety, including $3.0 \%$ and $8.1 \%$ with extremely severe depression and anxiety, respectively. Students not living with immediate family had higher depression score; female students and those in Preclinical Year 1 and Clinical Year 1 showed higher anxiety scores. Dysfunctional coping strategies and lower resilience are predictors of higher depression and anxiety symptoms.

Conclusions: Students show different levels of depressive and anxiety symptoms, signifying different levels of mental health support needs from universal mental health promotion to psychiatric treatment. Prevention programs can be targeted towards students with risk factors, such as not living with immediate family, undergoing first year of preclinical studies or clinical rotations, coping with dysfunctional strategies, and having low resilience. Additionally, medical educators must be aware of other, non-student factors that may impact student mental health, such as curriculum design and learning experience.
\end{abstract}

Keywords: Anxiety, Coping strategy, Depression, Undergraduate medical education, Medical students, Resilience

*Correspondence: adhitsr@gmail.com

Department of Psychiatry, Faculty of Medicine Universitas Indonesia -

Cipto Mangunkusumo Hospital, Jakarta, Indonesia

\section{Background}

The medical education system is designed to equip students with the knowledge and skills to provide safe, high-quality medical care within the framework of a 
healthcare system [1]. In the process, students form their identity as physicians that conform to professional and legal standards [2]. Thus, the time spent in medical education can be an exciting period of personal and professional development.

Students' physical and psychological well-being has been recognized as an important component in achieving those educational goals [3]. However, medical students face a myriad of challenges during their education that may pose a risk to their psychological well-being [4-6]. These challenges may arise from the medical education curriculum itself. Mastering all the required knowledge and skills in a relatively short period can lead to high academic workload, which may overwhelm students [4-6]. Additionally, parts of the workload may be deemed unnecessary or unrealistic [6]. The delivery of the curriculum, in the form of teaching and evaluation methods, can impact students' psychological health as well $[7,8]$. Students are also expected to adapt to different educational demands and settings, which become more pronounced when they undergo clinical rotations [5]. The hidden curriculum, composed of unwritten values, expectations, and power dynamics, can be another source of stress, especially when it exposes students to ethical conflicts [5]. Outright bullying or harassment against students remais prevalent too [5]. These stressors at school add to the stress from students' personal life. On the other hand, medical students are often deprived of their social support network and opportunities to recuperate $[4,6]$.

Consequently, medical students often experience poor mental health, including symptoms of depression and anxiety. Two meta-analyses have found that, globally, more than 1 in 4 students report symptoms of depression, and up to 1 in 10 students report suicidal ideation $[9,10]$. Another global meta-analysis found that 1 in 3 medical students experience symptoms of anxiety [11]. Longitudinal studies have revealed that students enter medical education with psychological well-being that is comparable or even better than the general population. However, their rates of depression and anxiety increase during their time in medical school $[12,13]$.

Mental health issues of medical students have gained significant attention, not only due to their prevalence, but also due to the short- and long-term consequences. Poor mental health may contribute to impaired academic performance and achievement of competencies [14]. It may also erode students' empathy, ethics, and other humanistic qualities that are essential for medical practice [15, 16]. Furthermore, there are concerns that untreated mental health issues in medical students are the underlying cause of the poor mental health of physicians [17]. Ultimately, the mental health of current medical students will, through multiple pathways, shape the healthcare system of the future.

Coping strategy and resilience are two characteristics that are thought to moderate the influence of stressors on student mental health. Students may employ different coping strategies when dealing with different demands during their education. If the coping strategy is effective and suitable for the demands at hand and the available resources, it can confer protection. On the other hand, ineffective or maladaptive coping may be associated with negative mental health outcomes $[18,19]$. Resilience is the ability to recover and grow from adversity through positive adaptation, without developing physical, psychological, or psychosocial disabilities [20, 21]. Resilient students can perceive problems as opportunities for growth, recognize available resources, organize themselves, and show creativity, optimism, and humour [20]. Furthermore, these two factors are of special interest for medical educators, as they are relatively amenable to psychological interventions to promote students' mental health, compared to the rigid structure of curriculum and medical training $[22,23]$.

As reflected by the proliferation of research on this topic, the importance of medical students' mental health has been widely recognized by medical educators. However, data from Indonesian setting is rather limited, which negatively affects how medical schools can respond to students' mental health needs. While the Indonesian medical education system may share some characteristics with those of other countries, there are also considerable differences. For example, the education to be a general physician is taken at the undergraduate level, while specialist residencies are considered as optional post-graduate study. Indonesia also has its own set of expected physician competencies, as influenced national health policies and resources, and medical education culture that may affect the mental health of students. Reflecting Indonesian cultures in general, Indonesian medical education culture has been described as collectivist and hierarchical, with high power distance and emphasis on connectedness, which can influence how students experience their medical education. While this situation may be familiar in Asian countries, it is less so in other regions [24]. As a result, mental health programs that are successful elsewhere cannot be simply transplanted to Indonesian context.

To fill that information gap, this study aims to estimate the prevalence of depressive and anxiety symptoms among medical students in Indonesia. Coping strategy and resilience are also explored as possible factors that contribute to students' mental health. Maladaptive coping strategies and lower resilience are hypothesized to be predictors of higher depressive and anxiety symptoms. 
The results of this study will give a picture of medical students' mental health and their needs for support. Moreover, the findings of this study will add to the geographical diversity of studies on medical student mental health worldwide and provide an empirical foundation for programs to protect and improve the mental health of Indonesian medical students.

\section{Methods}

\section{Context}

This study was conducted in a public institution, the Faculty of Medicine Universitas Indonesia (FKUI), Jakarta. The medical doctor study program is undertaken at the undergraduate level for at least 11 semesters: 7 semesters of preclinical studies leading to Bachelor of Medical Science degree, and 4 semesters of clinical rotations which ends with the Medical Doctor degree. The preclinical phase is arranged as system-based modules. Students also get early exposure to clinical experience and field work. The clinical rotations are mainly conducted in Cipto Mangunkusumo Hospital (RSCM), the national referral hospital in Indonesia, along with other referral hospitals and primary healthcare centers around the city. In the first year of clinical phase, students undergo rotations in 4 10-weeks 'major' rotations in internal medicine, pediatrics, obstetrics and gynecology, and surgery. Afterwards, they rotate in 7 4-weeks 'minor' rotations. Students graduate after passing the national physician competency examination, composed of computer-based multiple-choice examination and objective structured clinical examination (OSCE).

FKUI also runs an international class program. The program curriculum is similar to the regular class, but English is used as language of instruction and there is an additional year between the end of preclinical studies and clinical rotations. During that year, students conduct research in partner universities in Melbourne, Australia or Newcastle, England to earn a degree from their respective institutions.

\section{Design}

This cross-sectional study was conducted in the Faculty of Medicine Universitas Indonesia during the 2019-2020 academic year. The sample was selected using stratified random sampling, based on a complete list of students from the regular and international classes provided by faculty administration. For the regular class, 75 students were randomly selected from each year (Preclinical Years 1-4 and Clinical Years 1-2). Due to the smaller population of the international class, 80 students were randomized from preclinical phase (Preclinical Years 1-4) and another 80 students from the clinical phase (Clinical Years 1-2). Each selected student was given a written explanation about the nature and purpose of this study and were invited to participate. Those who respond to the invitation gave their written informed consent and filled the study questionnaire. Data collection had been completed before COVID-19 was declared a pandemic by the World Health Organization and the first case was found in the country.

The protocol of this study has been reviewed and given ethical clearance number ND-930/UN2.F1/ETIK/ PPM.00.02/2019 by the Health Research Ethics Committee of FKUI-RSCM. All methods in this study were carried out in accordance to relevant guidelines and regulations.

\section{Measurements}

The study questionnaire included questions on respondents' sociodemographic characteristics and self-report instruments measuring symptoms of depression and anxiety, coping strategy, and resilience. The sociodemographic variables include age, gender, region of origin, accommodation during education, financing, type of high school, study program, and year of study.

Symptoms of depression and anxiety were measured by their respective scales in the Depression Anxiety Stress Scale (DASS). DASS had been adapted into Bahasa Indonesia with adequate validity and reliability [25]. The depression and anxiety scales each have 14 items, and respondents score them 0 to 3 according to their degree of agreement. The sum represents the severity of symptoms. DASS was designed to be analyzed numerically [26]. Nevertheless, previous studies have used categorization. For depression, scores 0 to 9 is normal, 10 to 13 mild, 14 to 20 moderate, 21 to 27 severe, and 28 or more is extremely severe. For depression, scores 0 to 7 is normal, 8 to 9 mild, 10 to 14 moderate, 15 to 19 severe, and 20 or more is extremely severe [12].

Coping strategy was measured using Brief COPE (Coping Orientation to Problems Experienced), which consists of 14 subscales for different coping strategies: active coping, planning, positive reframing, acceptance, humor, religion, using emotional support, using instrumental support, self-distraction, denial, venting, substance use, behavioral disengagement, and self-blame. The instrument had been adapted and validated for Indonesian population [27]. Each subscale consists of 2 items, scored between 0 to 3 . Higher score on a subscale represents more frequent utilization of that coping strategy [28]. The 14 subscales can be grouped into problem-focused coping, emotion-focused coping, and dysfunctional coping [29]. Students were instructed to fill the Brief COPE according to their coping strategy when facing challenges or issues related to their medical education. 
Last, resilience is measured using Connor-Davidson Resilience Scale (CD-RISC). The instrument is composed of 25 items, each scored between 0 to 4 . Students with higher sum of all items are said to have higher resilience [30]. It had been translated into Bahasa Indonesia with adequate validity and reliability [31].

\section{Data analyses}

Study data was managed using SPSS for Windows version 22. Descriptive statistics for categorical variables are reported as proportion. For numerical variables, the Kolmogorov-Smirnov test was used to assess distribution of data. Variables that follow normal distribution are reported as mean with standard deviation (SD) while variables that do not are reported as median with interquartile range (IQR). Comparison of depressive and anxious symptoms between two groups was carried out using unpaired t-test or Mann-Whitney test; whereas comparison between more than two groups was done by ANOVA or Kruskal-Wallis test. The relationship between symptoms of depression, symptoms of anxiety, coping strategy, and resilience was measured by correlation, reported as Pearson correlation coefficient or Spearman correlation coefficient. The level of statistical significance was set at $p<0.05$. Subsequently, we performed multiple linear regression analyses to identify predictors of depression and anxiety symptoms by including variables with $p$-value $<0.2$ in the bivariate analyses, and for correlations, a correlation coefficient of $r>0.2$.

\section{Results}

Out of the 610 randomly selected students, 532 responded (87,2\% response rate) and filled the study questionnaire: 343 students from preclinical phase and 189 from clinical phase. The remaining selected students did not respond to the invitation to participate. The age of respondents ranges from 17 to 27 years old, with a mean of $20.65(S D=1,98)$. More than half $(59.6 \%)$ of respondents is female. More than two-thirds come from the Greater Jakarta Area, and 44.2\% do not live with their immediate family. Approximately $61.7 \%$ studied in public high school. Almost all respondents finance their studies independently without any financial aid.

Approximately $22.2 \%$ of students showed depressive symptoms, mostly in the mild and moderate category, with a median score of 4 (IQR $1-9)$. Meanwhile, symptoms of anxiety were reported by $48.1 \%$ of students. Similarly, most are in the mild and moderate range, with a median score of 7 (IQR 4 - 13) (Table 1). Among all students, $18,6 \%$ showed symptoms of both depression and anxiety. The scores for depressive and anxiety symptoms exhibit statistically significant correlation with moderate strength $(r=0.653 ; p<0.001)$.
Table 1 Distribution of depressive and anxiety symptoms

\begin{tabular}{llllll}
\hline & \multicolumn{2}{l}{ Depression } & & \multicolumn{2}{l}{ Anxiety } \\
& $\mathbf{n}$ & & & $\mathbf{n}$ & $\%$ \\
\hline Normal & 414 & 77.8 & & 276 & 51.9 \\
Mild & 47 & 8.8 & & 64 & 12.0 \\
Moderate & 46 & 8.6 & & 98 & 18.4 \\
Severe & 9 & 1.7 & & 51 & 9.6 \\
Extremely severe & 16 & 3.0 & & 43 & 8.1 \\
\hline
\end{tabular}

Students not living with their immediate family had higher score of depressive symptoms. Higher scores of anxiety symptoms were found in female students and students of different years, especially in the first year of preclinical studies and in the first year of clinical rotations (Table 2).

Students mainly utilize problem-focused and emotionfocused coping strategies, without any particular strategy being used more frequently. Most show statistically significant association with symptoms of depression, but the strength of correlation is low. Only 'using emotional support' has a relatively higher correlation coefficient $(r=-0.291 ; p<0.001)$ (Table 3).

More variation is found in the dysfunctional coping strategies. Students use self-distraction, venting, and self-blame at a similar rate to problem-focused and emotion-focused coping. Less commonly employed coping strategies are denial, behavioral disengagement, and substance use. As a group, dysfunctional coping is significantly correlated with both symptoms of depression $(r=0.461 ; p<0.001)$ and anxiety $(r=0.378 ; p<0.001)$. For specific coping strategies, the strongest correlations with depressive and anxious symptoms are shown by selfblame and behavioral disengagement. Self-distraction has a significant but low-strength correlation, while venting is not correlated to either depressive symptoms or anxious symptoms (Table 3 ).

The median score of resilience, as measured by CDRISC, is 68 (IQR 58 - 77). Higher resilience is moderately correlated with lower scores of depressive symptoms $(r=-0.428 ; p<0.001)$ and anxious symptoms $(r=-0.298$; $p<0.001$ ) (Table 3).

We conducted multiple linear regression analysis for score of depressive symptoms with the following independent variables: accommodation, study program, secondary education, resilience, and the coping strategies emotional support, denial, substance use, behavioral disengagement, and self-blame. No significant collinearity was observed among the independent variables, with tolerance $>0.4$ and variance inflation factor $<2$. The model is statistically significant $(p<0.001 ; F=47.626)$ and showed an adjusted $R^{2}$ of $44,1 \%$. The variables study program and 
Table 2 Distribution of depressive and anxiety symptoms by sociodemographic variables

\begin{tabular}{|c|c|c|c|c|c|c|}
\hline & $n$ & (\%) & $\begin{array}{l}\text { Depression score } \\
\text { (median [IQR]) }\end{array}$ & pvalue & $\begin{array}{l}\text { Anxiety score } \\
\text { (median [IQR]) }\end{array}$ & $p$ value \\
\hline All subjects & 532 & 100 & $4(1-9)$ & - & $7(4-13)$ & - \\
\hline \multicolumn{7}{|l|}{ Gender } \\
\hline Male & 215 & 40.4 & $4(1-8)$ & \multirow[t]{2}{*}{0.707} & $7(4-11)$ & \multirow[t]{2}{*}{0.027} \\
\hline Female & 317 & 59.6 & $4(1-9)$ & & $8(4-13)$ & \\
\hline \multicolumn{7}{|l|}{ Region of origin } \\
\hline Greater Jakarta & 366 & 68.8 & $4(1-9)$ & \multirow[t]{3}{*}{0.862} & $7(4-12)$ & \multirow[t]{3}{*}{0.168} \\
\hline Java outside Greater Jakarta & 96 & 18.0 & $3(3-8)$ & & $7(3-11)$ & \\
\hline Other than Java & 70 & 13.2 & $4(1.75-9)$ & & $8(4-15)$ & \\
\hline \multicolumn{7}{|l|}{ Accommodation } \\
\hline With immediate family & 297 & 55.8 & $3(1-8)$ & \multirow[t]{2}{*}{0.041} & $7(4-12)$ & \multirow[t]{2}{*}{0.580} \\
\hline Not with immediate family & 235 & 44.2 & $4(2-9)$ & & $7(4-13)$ & \\
\hline \multicolumn{7}{|l|}{ Financing } \\
\hline Out-of-pocket & 479 & 90.0 & $4(1-9)$ & \multirow[t]{2}{*}{0.716} & $7(4-12)$ & \multirow[t]{2}{*}{0.799} \\
\hline Partial or full scholarship & 53 & 10.0 & $3(2-10.5)$ & & $7(3-13,5)$ & \\
\hline \multicolumn{7}{|l|}{ Secondary education } \\
\hline Public high school & 328 & 61.7 & $4(1-8)$ & \multirow[t]{3}{*}{0.141} & $7(4-12)$ & \multirow[t]{3}{*}{0.324} \\
\hline Private high school & 159 & 29.9 & $5(2-10)$ & & $8(4-13)$ & \\
\hline International high school & 45 & 8.5 & $3(1-7)$ & & $6(4-10,5)$ & \\
\hline \multicolumn{7}{|l|}{ Study program } \\
\hline Regular class & 376 & 70.7 & $4(2-9.75)$ & \multirow[t]{2}{*}{0.099} & $7(4-13)$ & \multirow[t]{2}{*}{0.220} \\
\hline International class & 156 & 29.3 & $3(1-8)$ & & $7(4-11)$ & \\
\hline \multicolumn{7}{|l|}{ Stage of study } \\
\hline Preclinical & 343 & 64.5 & $4(1-9)$ & \multirow[t]{2}{*}{0.865} & $7(4-13)$ & \multirow[t]{2}{*}{0.308} \\
\hline Clinical & 189 & 35.5 & $4(1-8)$ & & $7(3-11)$ & \\
\hline \multicolumn{7}{|l|}{ Year of study } \\
\hline Preclinical Year 1 & 90 & 16.9 & $5(2-9.25)$ & \multirow[t]{6}{*}{0.441} & $10(6-16)$ & \multirow[t]{6}{*}{$<0.001$} \\
\hline Preclinical Year 2 & 79 & 14.8 & $4(2-9)$ & & $8(4-13)$ & \\
\hline Preclinical Year 3 & 92 & 17.3 & $3(1-7.75)$ & & $6(4-10)$ & \\
\hline Preclinical Year 4 & 82 & 15.4 & $3(2-9)$ & & $6(4-11)$ & \\
\hline Clinical Year 1 & 93 & 17.5 & $5(1-10)$ & & $8(3.5-14)$ & \\
\hline Clinical Year 2 & 93 & 18.0 & $3(1-7)$ & & $7(3-10)$ & \\
\hline
\end{tabular}

Mann-Whitney U Test for comparison between 2 groups and Kruskal-Wallis Test for comparison between more than 2 groups; statistically significant results ( $p<0.05$ ) printed in bold

secondary education did not reach statistical significance. Resilience and seeking emotional support are associated with lower scores of depression, while substance use, self-blame, denial, behavioral disengagement, and not living with family increase the score (Table 4).

For symptoms of anxiety, the multiple linear regression analysis included the independent variables gender, region of origin, year of study, resilience, and coping strategies denial, substance use, behavioral disengagement, and self-blame. There was no significant collinearity among the independent variables. The resulting model was significant $(p<0.001 ; F=31.755)$ and has an adjusted $R^{2}$ of $31,7 \%$. Along with female gender and originating from outside Jakarta, the coping strategies substance use, self-blame, denial, and behavioral disengagement predict higher anxiety; while resilience and increasing year of study are associated with lower anxiety scores. (Table 4).

\section{Discussion}

The prevalence of depressive symptoms in this study at $22.2 \%$ is lower than the results of two global meta-analysis that each found the prevalence at $27.2 \%$ and $28 \%$ as well as the results of subgroup analysis from Asian countries $(29.1 \%$ and $30.1 \%)[9,10]$. It is also lower compared to 3 studies using the same instrument and cut-off score (40.4\%) [9]. Students not living with their immediate family have higher symptoms of depression. This finding may point to the loss of social support from family members 
Table 3 Distribution of resilience and coping strategy, and their correlations with depressive and anxiety symptoms

\begin{tabular}{|c|c|c|c|c|c|}
\hline & \multirow[t]{2}{*}{ Median (IQR) } & \multicolumn{2}{|c|}{ Depression } & \multicolumn{2}{|c|}{ Anxiety } \\
\hline & & $r$ & pvalue & $r$ & pvalue \\
\hline Resilience (0-100) & $68(58-77)$ & -0.428 & $<0.001$ & -0.298 & $<0.001$ \\
\hline \multicolumn{6}{|l|}{ Coping strategy $(0-6)$} \\
\hline $\begin{array}{l}\text { Problem-focused } \\
(0-18)\end{array}$ & $12(11-14)$ & -0.188 & $<0.001$ & -0.008 & 0.851 \\
\hline Active coping & $4(4-5)$ & -0.160 & $<0.001$ & -0.014 & 0.755 \\
\hline Planning & $4(4-5)$ & -0.094 & 0.030 & -0.002 & 0.958 \\
\hline $\begin{array}{l}\text { Instrumental sup- } \\
\text { port }\end{array}$ & $4(3-5)$ & -0.172 & $<0.001$ & -0.009 & 0.840 \\
\hline $\begin{array}{l}\text { Emotion-focused } \\
(0-30)\end{array}$ & $20(17-22)$ & -0.123 & 0.005 & -0.017 & 0.692 \\
\hline Acceptance & $4(4-5)$ & 0.000 & 0.996 & -0.019 & 0.655 \\
\hline Humor & $3(2-4)$ & 0.177 & $<0.001$ & 0.054 & 0.211 \\
\hline Religion & $4(3-5)$ & -0.131 & 0.002 & 0.021 & 0.621 \\
\hline Emotional support & $4(3-5)$ & -0.291 & $<0.001$ & -0.104 & 0.017 \\
\hline Positive reframing & $4(4-5)$ & -0.169 & $<0.001$ & -0.090 & 0.039 \\
\hline Dysfunctional $(0-36)$ & $14(11-16)$ & 0.461 & $<0.001$ & 0.378 & $<0.001$ \\
\hline Self-distraction & $4(4-5)$ & 0.115 & 0.008 & 0.084 & 0.051 \\
\hline Denial & $1(0-2)$ & 0.268 & $<0.001$ & 0.230 & $<0.001$ \\
\hline Venting & $3(2-4)$ & -0.006 & 0.885 & 0.008 & 0.849 \\
\hline Substance use & $0(0-0)$ & 0.228 & $<0.001$ & 0.201 & $<0.001$ \\
\hline $\begin{array}{l}\text { Behavioral disen- } \\
\text { gagement }\end{array}$ & $1(0-2)$ & 0.414 & $<0.001$ & 0.311 & $<0.001$ \\
\hline Self-blame & $3(2-5)$ & 0.438 & $<0.001$ & 0.378 & $<0.001$ \\
\hline
\end{tabular}

Correlation shown using Spearman's rank correlation coefficient; statistically significant results $(p<0.05)$ printed in bold

that has been known to increase the risk of depression $[32,33]$. Meanwhile, some other studies did not find similar association [34, 35]. Students may compensate the loss of support from immediate family through other sources of social support, such as peer groups and counsellors [32].

Symptoms of anxiety were found in $48.1 \%$ of students, much higher than the $33.8 \%$ prevalence from a global meta-analysis, and still higher compared to regions with the highest prevalence of anxiety: the Middle East (42.4\%) and Asia (35.2\%) [11]. The same meta-analysis also found a wide range of prevalence from studies using DASS, from $12.8 \%$ to $78.4 \%$ [11]. Female students showed higher scores of anxiety, which may reflect the difference of prevalence in the general population [36]. While the meta-analysis in medical students did not find the same association, a study from Brazil also found that female students have higher scores of state-anxiety and traitanxiety [34]. Previous research has pointed at various possible causes for this sex difference in the prevalence of anxiety. Biological factors may include hormonal profile, neural circuitry, and neurotransmitter activity [37, 38].
These factors may also underlie psychological characteristics that predisposes to anxiety, such as neuroticism, ruminative tendencies, higher interpersonal sensitivity and attentional bias toward threats [37, 39, 40]. Women also face different life experiences and social expectations, which shape their brain and psychological development and, ultimately, their response to stressors [37, 40].

Higher scores of anxiety symptoms are found among students in the first year of preclinical studies and the first year of clinical rotations. Those years may represent periods of adaptation, respectively from secondary to tertiary education and from preclinical to clinical stage of medical education. They have very different learning activities and expectations, as well as educational environments with each of their written and unwritten rules. This pressure to adapt may become a considerable stressor for some students [5].

Respondents of this study show moderate level of resilience (68 [58-77]), slightly higher than the results of another study conducted in FKUI (64.76 to 67.60) [41] and also higher than a study from 3 medical schools in China (61.69 [SD = 10.55]) [42]. Higher resilience is moderately correlated with lower scores of both depression and anxiety symptoms. Existing studies have established similar correlations, albeit with different strengths of correlation [43-45]. More severe symptoms of depression and anxiety are more likely to be found among students with low resilience [45]. Resilience are also associated with better mental health indicators beyond depression and anxiety, such as quality of life, level of stress, burnout syndrome, and perception of educational environment. In many studies, resilience is conceptualized as a buffer or mediator between external stressors, student characteristics, and mental health outcomes [42-46].

Students employ problem-focused and emotionfocused coping strategies more frequently than dysfunctional coping. Among the dysfunctional coping strategies, only self-distraction is commonly used by students. This finding concurs with the results of another study from FKUI that only included first-year students [29]. Studies from other countries reveal similar trend of students relying on more adaptive or active coping methods such as acceptance, cognitive reappraisal, problem solving, planning, and seeking support $[47,48]$. However, a study revealed that students' use of distancing and selfcontrol (keeping things to oneself) increases as they progress through their medical education [47].

Dysfunctional coping is clearly correlated with higher symptoms of depression and anxiety, with behavioral disengagement and self-blame showing the strongest association. Another study found that behavioral disengagement is the coping method that is more specifically used among students with mental health issues [48]. 
Table 4 Multiple linear regression analysis for scores of depressive and anxiety symptoms

\begin{tabular}{|c|c|c|c|c|c|}
\hline Variable & Unstandardized $\beta(95 \% \mathrm{Cl})$ & SE & Standardized $\beta$ & $t$ & $p$ value \\
\hline \multicolumn{6}{|c|}{ Dependent variable: score of depressive symptoms } \\
\hline Constant & $7.226(3.352 ; 11.100)$ & 1.972 & & 3.665 & $<0.001$ \\
\hline Study program & $-0.740(-1.829 ; 0.349)$ & 0.554 & -0.048 & -1.334 & 0.183 \\
\hline Secondary education & $-0.027(-0.794 ; 0.740)$ & 0.390 & -0.002 & -0.069 & 0.945 \\
\hline Accommodation & $1.410(0.469 ; 2.324)$ & 0.465 & 0.099 & 3.031 & 0.003 \\
\hline Resilience & $-0.089(-0.129 ;-0.049)$ & 0.020 & -0.174 & -4.375 & $<0.001$ \\
\hline Coping: emotional support & $-0.764(-1.102 ;-0.426)$ & 0.172 & -0.152 & -4.443 & $<0.001$ \\
\hline Coping: substance use & $1.193(0.744 ; 1.643)$ & 0.229 & 0.175 & 5.215 & $<0.001$ \\
\hline Coping: denial & $0.455(0.024 ; 0.885)$ & 0.219 & 0.072 & 2.073 & 0.039 \\
\hline Coping: behavioral disengagement & $1.636(1.161 ; 2.112)$ & 0.242 & 0.278 & 6.765 & $<0.001$ \\
\hline Coping: self-blame & $1.211(0.888 ; 1.534)$ & 0.164 & 0.256 & 7.365 & $<0.001$ \\
\hline \multicolumn{6}{|c|}{ Dependent variable: score of anxiety symptoms } \\
\hline Constant & $6.076(2.304 ; 9.848)$ & 1.920 & & 3.165 & 0.002 \\
\hline Gender & $1.627(0.695 ; 2.559)$ & 0.474 & 0.124 & 3.429 & 0.001 \\
\hline Region of origin & $1.027(0.384 ; 1.669)$ & 0.327 & 0.114 & 3.138 & 0.002 \\
\hline Year of study & $-0.490(-0.755 ;-0.225)$ & 0.135 & -0.132 & -3.626 & $<0.001$ \\
\hline Resilience & $-0.081(-0.121 ;-0.042)$ & 0.020 & -0.174 & -4.104 & $<0.001$ \\
\hline Coping: substance use & $1.395(0.944 ; 1.846)$ & 0.230 & 0.224 & 6.078 & $<0.001$ \\
\hline Coping: denial & $0.769(0.334 ; 1.204)$ & 0.221 & 0.134 & 3.473 & 0.001 \\
\hline Coping: behavioral disengagement & $0.723(0.244 ; 1.202)$ & 0.244 & 0.135 & 2.966 & 0.003 \\
\hline Coping: self-blame & $1.035(0.709 ; 1.360)$ & 0.166 & 0.240 & 6.249 & $<0.001$ \\
\hline
\end{tabular}

Cl confidence interval; SE standard error; variables with $p<0.05$ printed in bold

Students that employ 'avoidant' strategies (substance use, emotional distancing, self-harm) more frequently than or as frequently as 'approach' strategies (seeking help, seeking support, increasing effort) are more likely to have higher depression symptoms and to be burned out [32]. In contrast to previous studies, almost none of the respondents in this study resort to substance use as a coping strategy. This is a positive finding because besides being a sign of dysfunctional coping, substance use among medical students can be a risk factor of poor mental health and may compromise academic performance as well as patient safety $[49,50]$.

The results of this study showed that the respondents use various strategies in the problem-focused and emotion-focused coping groups with similar frequency. Individuals with a wide range of available coping strategies are said to possess a broad coping repertoire. It also hinted that students do not show a rigid preference for any particular coping strategy. In other words, they have a balanced coping profile [51]. A study in Singaporean medical students reveal that they are able to employ different coping strategies to manage different academic or relationship demands during the course of their medical education. They are also capable of judging whether certain coping strategies are effective or whether they should try alternative approaches [18]. Broad coping repertoire and balanced profile, coupled with the ability to accurately judge the effectiveness of a coping strategy, mean that students have the coping flexibility to match their strategy with the demands at hand [51]. Some studies suggest that coping flexibility is a stronger predictor of mental health compared to individual coping strategies $[51,52]$. A study found that students in the clinical stage of medical education show more appreciation of the relationship between coping, stress, and mental health issues. Their understanding is also more grounded in real-life situations, instead of the more theoretical perspective of preclinical students [19].

In general, this study found that depression and anxiety are significantly correlated to resilience and coping strategy. However, the strength of correlations is in the low to moderate range, while the multiple linear regression analysis models can only explain less than half of the variance in outcome $(44,1 \%$ and $31,7 \%$ for depression and anxiety scores, respectively). This finding can be explained by the fact that mental health depends on the complex interactions between countless internal and external factors, only some of which was explored in this study. In the lens of biopsychosocial model, students as individuals with their unique biological and psychological characteristics undergo the long process of medical education that exerts considerable pressure, 
which can be moderated by social support or exacerbated by the lack of social resources [53]. It is highly unlikely that any single factor can have a major impact. Instead, it is more likely that all the different factors influence mental health with smaller effects through different pathways. Along this vein, Dunn et al. offered the concept of a "coping reservoir" for medical student well-being. Student's characteristics determine the internal structure of the reservoir, while negative and positive experiences can respectively deplete or replenish the reservoir. The interaction of those experiences and the reservoir itself shapes the outcome of student's mental health [4].

The prevalence of depressive and anxiety symptoms in this study points to different levels of mental health support needs that should be anticipated by stakeholders. The majority of students have normal to low scores of depression and anxiety. However, it should be noted that low level or sub-threshold symptoms are significant risk factors for full-threshold mental disorder in the future. The risk is even more pronounced in late teenagers and young adults, the age range of respondents in this study $[54,55]$. Additionally, those with subthreshold symptoms may already experience impairment in their role functioning and require professional help $[55,56]$. Students without symptoms may also have risk factors for mental health issues, such as low resilience and a tendency for dysfunctional coping as found in this study. Therefore, it is important that medical schools regularly screen all students for mental health symptoms and risk factors. Results of screening can help schools formulate suitable mental health promotion programs and identify at-risk students who need more selective prevention programs [57]. For example, specific programs can be designed for students entering their very first year in medical school or those transitioning into clinical rotations.

Different types of mental health promotion and prevention programs can be found in the literature, as schools start appreciating the importance of mental health. A survey of medical schools in the United States found that more than half offer student well-being curriculum, with mandatory and optional activities. They may include peer support, stress management, counselling, physical activities, financial preparedness, recreational activities, and social events [58]. The design of wellness programs should center the lived experience and actual needs of students to ensure that the programs are effective and acceptable, and that resources are well-spent. Students and faculty may have different perspectives on well-being and ways to promote it [22]. Nevertheless, students are capable of identifying their needs and specify their preferred programs, or even generate their own ideas for mental health programs $[59,60]$.
Last, students who are experiencing significant symptoms of depression and anxiety should be provided access to effective and safe psychiatric treatment. An evaluation of mental health services for medical students in University of Cambridge found that such dedicated specialist service can effectively improve psychological well-being and functioning. Students also expressed their appreciation for the flexibility and accessibility of the service [59]. Barriers to access must be addressed, especially the stigma surrounding medical students and mental health issues [61].

The finding that mental health issues are correlated to resilience and coping strategy, both "internal" factors of the student, does not put the burden of mental health solely on students. Students are deeply affected by the social and educational systems they inhabit, over which they have little to no influence. Their "internal" factors, including resilience and coping, are also shaped by available resources and social environment. Thus, policy makers have a moral and ethical responsibility as well as the privilege to promote students' mental health $[62,63]$.

Programs that are aimed to help students cope with the pressure of medical education can be beneficial. However, they exist in a paradigm in which that distress in medical education is a given and that medical schools may help students by "strengthening" them. Policy makers at all levels will eventually need to address the sources of distress in the education process itself if they wish to have a mental health program that is truly preventive [7]. A qualitative study that allowed students to generate their own ideas for well-being programs reported that students want changes to how their education is carried out [60]. Some suggested changes to the curriculum include pass/fail grading system, reducing contact hours, evaluating the depth of learning materials, and reformulating feedback given to students [7]. A systematic review found that, while better quality studies are needed, these interventions do promote students' mental health [64].

This study is one of the few that have systematically assessed mental health issues among Indonesian medical students and possible risk factors. While studies from other countries may inform Indonesian medical education system, locally-produced studies are important to uncover the similarities and differences that can assist in adopting best practices into Indonesian context. Furthermore, the sample size of this study is relatively large and representative, as subjects are selected through probabilistic methods from each year of medical school.

On the other hand, the cross-sectional design of this study prevents us from making definitive conclusions about the direction of causality between symptoms and risk factors. For example, it is possible that depressive symptoms reinforce dysfunctional coping strategies, 
such as self-blame and behavioral disengagement, that are included among commonly used criteria for depressive disorders [65]. The timing of measurement may also contribute to the variation of symptom severity [66]. Lastly, this study may have limited generalizability to the whole medical student population in Indonesia as the sample was taken from only one medical school in Jakarta, out of more than 70 medical schools in the country. Further studies would need bigger, more representative samples to gain a better understanding of mental health issues among Indonesian medical students.

Future studies could utilize cohort design to analyze the fluctuations of depressive and anxiety symptoms, as well as dynamic risk factors, over time in medical school. That information would enable researchers to determine the presence of causality and its direction. Such studies could be integrated into the medical education system at each school. Baseline data can be collected at student intake, and then followed up with regular frequency until students graduate or even beyond graduation. They could also consider additional risk factors, including sociocultural factors. In light of the COVID-19 pandemic, this study provides data on Indonesian medical students' mental health prior to the pandemic itself. While this study does not reflect current situation in the ongoing pandemic, it can serve as baseline data to evaluate its impact.

\section{Conclusions}

Approximately 1 in 4 students report experiencing symptoms of depression, while almost 1 in 2 report symptoms of anxiety. Dysfunctional coping strategies, especially self-blame and behavioral disengagement, are predictors of depression and anxiety symptoms. Students with higher resilience experience fewer depressive and anxiety symptoms. Medical educators and policy makers need to anticipate the different levels of support that students may need, from mental health promotion, prevention of mental health issues, to specialist psychiatric treatment. Beyond interventions that target students, educators must also evaluate and make changes to the education system itself to reduce the risk of mental health issues.

\section{Abbreviations}

FKUI: Faculty of Medicine Universitas Indonesia; RSCM: Cipto Mangunkusumo Hospital; DASS: Depression Anxiety Stress Scale; CD-RISC: Connor-Davidson Resilience Scale; IQR: Interquartile range; SD: Standard deviation.

\section{Acknowledgements}

The authors would like to express our gratitude to all the students who participated in this study, and to the Faculty of Medicine Universitas Indonesia and its undergraduate coordinators for their support throughout the study.

\section{Authors' contributions}

ASR conceived the study, collected study data, performed data analysis, and drafted the initial version of this manuscript. IK, FA, and NWR made substantial contributions to the study design, interpretation of study data, substantial manuscript revision. All authors have approved the submitted manuscript and are accountable for the accuracy and integrity of the content.

\section{Funding}

This research received no external funding.

\section{Availability of data and materials}

The datasets used and/or analysed in this study are available from the corresponding author on reasonable request.

\section{Declarations}

\section{Ethical approval and consent to participate.}

All methods in this study were conducted in accordance with relevant guidelines and regulations. The study protocol has been approved by the Health Research Ethics Committee of Faculty of Medicine Universitas Indonesia Cipto Mangunkusumo Hospital (ethical clearance number ND-930/UN2.F1/ ETIK/PPM.00.02/2019). Prior to data collection, all participants were provided with information about this study, including their voluntary participation and data confidentiality, and gave their written informed consent.

\section{Consent for Publication}

Not applicable - this manuscript does not publish personal data of study participants.

\section{Competing Interests}

The authors declare that they have no competing interests.

Received: 12 August 2021 Accepted: 31 January 2022

Published online: 07 February 2022

References

1. Sklar DP, Hemmer PA, Durning SJ. Medical education and health care delivery: A call to better align goals and purposes. Acad Med. 2018;93(3):384-90.

2. Irby DM, Hamstra SJ. Parting the clouds: three professionalism frameworks in medical education. Acad Med. 2016;91(12):1606-11. Available from: (http://www.ncbi.nlm.nih.gov/pubmed/27119331).

3. Kemp S, Hu W, Bishop J, Forrest K, Hudson JN, Wilson I, et al. Medical student wellbeing - A consensus statement from Australia and New Zealand. BMC Med Educ. 2019;19:69.

4. Dunn L, Iglewicz A, Moutier C. A conceptual model of medical student well-being: promoting resilience and preventing burnout. Acad Psychiatry. 2008;32(1):44-53. Available from: (http://link.springer.com/article/10. 1176/appi.ap.32.1.44)

5. Dyrbye LN, Thomas MR, Shanafelt TD. Medical student distress: causes, consequences, and proposed solutions. Mayo Clin Proc 2005;80(12):1613-22. Available from: (http://linkinghub.elsevier.com/retri eve/pii/S0025619611610574)

6. Hill MR, Goicochea S, Merlo LJ. In their own words: stressors facing medical students in the millennial generation. Med Educ Online. 2018;23:1530558. https://doi.org/10.1080/10872981.2018.1530558.

7. Slavin SJ, Schindler DL, Chibnall JT. Medical student mental health 3.0: Improving student wellness through curricular changes. Acad Med. 2014;89(4):573-7.

8. Lyndon MP, Strom JM, Alyami HM, Yu T-C, Wilson NC, Singh PP, et al. The relationship between academic assessment and psychological distress among medical students: a systematic review. Perspect Med Educ. 2014;3:405-18.

9. Rotenstein LS, Ramos MA, Torre M, Segal JB, Peluso MJ, Guille C, et al. Prevalence of depression, depressive symptoms, and suicidal ideation among medical students: a systematic review and meta-analysis. JAMA. 2016;316(21):2214-36. Available from: (http://jama.jamanetwork.com/ article.aspx?doi=10.1001/jama.2016.17324). 
10. Puthran R, Zhang MWB, Tam WW, Ho RC. Prevalence of depression amongst medical students: A meta-analysis. Med Educ. 2016;50(4):456-68.

11. Quek TTC, Tam WWS, Tran BX, Zhang M, Zhang Z, Ho CSH, et al. The global prevalence of anxiety among medical students: a meta-analysis. Int J Environ Res Public Health. 2019;16(15):2735.

12. Yusoff MSB, Abdul Rahim AF, Baba AA, Ismail SB, Mat Pa MN, Esa AR The impact of medical education on psychological health of students: a cohort study. Psychol Health Med. 2013;18(4):420-30. Available from: (http://www.tandfonline.com/doi/abs/10.1080/13548506.2012.740162).

13. Brazeau CMLR, Shanafelt T, Durning SJ, Massie FS, Eacker A, Moutier $C$, et al. Distress among matriculating medical students relative to the general population. Acad Med. 2014;89(11):1520-5.

14. Chandavarkar U, Azzam A, Mathews CA. Anxiety symptoms and perceived performance in medical students. Depress Anxiety. 2007:24:103-11

15. Fargen KM, Drolet BC, Philibert I. Unprofessional behaviors among tomorrow's physicians: Review of the literature with a focus on risk factors, temporal trends, and future directions. Acad Med. 2016;91 (6):858-64.

16. Park KH, Kim D, Kim SK, Yi YH, Jeong JH, Chae J, et al. The relationships between empathy, stress and social support among medical students. Int J Med Educ. 2015;6:103-8.

17. Dzau VJ, Kirch DG, Nasca TJ. To care is human — collectively confronting the clinician-burnout crisis. N Engl J Med. 2018;378(4):312-4. Available from: (http://www.nejm.org/doi/10.1056/NEJMp1715127).

18. Wu L, Farquhar J, Ma J, Vidyarthi AR. Understanding Singaporean medical students'stress and coping. Singapore Med J. 2018;59(4):172-6.

19. Farquhar J, Lie D, Chan A, Ow M, Vidyarthi A. Understanding medical students' experience with stress and its related constructs: a focus group study from Singapore. Acad Psychiatry. 2018;42(1):48-57. Available from: (http://link.springer.com/10.1007/s40596-017-0703-7).

20. Tempski P, Martins MA, Paro HBMS. Teaching and learning resilience: A new agenda in medical education. Med Educ. 2012;46(4):345-6.

21. de Oliveira ACP, Machado APG, Aranha RN. Identification of factors associated with resilience in medical students through a cross-sectional census. BMJ Open. 2017;7(11):e017189. Available from: (http://bmjopen.bmj. com/lookup/doi/10.1136/bmjopen-2017-017189).

22. Farquhar J, Kamei R, Vidyarthi A. Strategies for enhancing medical student resilience: student and faculty member perspectives. Int J Med Educ. 2018;9:1-6. Available from: (http://www.ijme.net/archive/9/strategies-toenhance-medical-student-resilience/).

23. Cheung EO, Kwok I, Ludwig AB, Burton W, Wang X, Basti N, et al. Development of a positive psychology program (LAVENDER) for preserving medical student well-being: a single-arm pilot study. Glob Adv Heal Med. 2021;10:216495612098848.

24. Soemantri D, Greviana N, Findyartini A, Azzahra TB, Suryoadji KA, Mustika $R$, et al. "To obey or not to obey" - Medical students' response towards professional dilemmas in a hierarchical and collectivist culture. PLoS One. 2021;16(12):e0261828. https://doi.org/10.1371/journal.pone.0261828.

25. Damanik ED. Pengujian reliabilitas, validitas, analisis item dan pembuatan norma Depression Anxiety Stress Scale (DASS): Berdasarkan penelitian pada kelompok sampel Yogyakarta dan Bantul yang mengalami gempa bumi dan kelompok sampel Jakarta dan sekitarnya yang tidak. Universitas Indonesia; 2006

26. UNSW Psychology. Depression Anxiety Stress Scales. Sydney: Psychology Foundation of Australia; 2014. [cited 2018 Mar 14] Available from: (http:// www2.psy.unsw.edu.au/dass/).

27. Setyorini SA. Hubungan antara individual coping, dyadic coping, dan kepuasan pernikahan pada penderita penyakit kronis. Universitas Indonesia; 2012.

28. Carver CS. You want to measure coping but your protocol's too long: consider the Brief COPE. Int J Behav Med. 1997:4(1):92-100.

29. Palupi R, Findyartini A. The relationship between gender and coping mechanisms with burnout events in first-year medical students. Korean J Med Educ. 2019;31(4):331-42.

30. Connor KM, Davidson JRT. Development of a new resilience scale: the Connor-Davidson Resilience Scale (CD-RISC). Depress Anxiety 2003;18:76-82.

31. Davidson JRT, Connor KM. Connor-Davidson Resilience Scale (CD-RISC) Manual. 2018. https://www.cd-risc.com.
32. Thompson G, McBride RB, Hosford CC, Halaas G. Resilience among medical students: The role of coping style and social support. Teach Learn Med. 2016:28(2):174-82.

33. Bore M, Kelly B, Nair B. Potential predictors of psychological distress and well-being in medical students: a cross-sectional pilot study. Adv Med Educ Pract. 2016;7:125-35.

34. Mayer FB, Santos IS, Silveira PSP, Lopes MHI, de Souza ARND, Campos EP, et al. Factors associated to depression and anxiety in medical students: a multicenter study. BMC Med Educ. 2016;16(1):282. Available from: (http:// bmcmededuc.biomedcentral.com/articles/10.1186/s12909-016-0791-1).

35. Bassols AM, Okabayashi LS, da Silva AB, Carneiro BB, Feijó F, Guimarães GC, et al. First- and last-year medical students: Is there a difference in the prevalence and intensity of anxiety and depressive symptoms? Rev Bras Psiquiatr. 2014;36(3):233-40.

36. Craske MG, Stein MB, Eley TC, Milad MR, Holmes A, Rapee RM, et al. Anxiety disorders. Nat Rev Dis Prim. 2017;3:17024. https://doi.org/10.1038/ nrdp.2017.24.

37. Altemus M, Sarvaiya N, Neill Epperson C. Sex differences in anxiety and depression clinical perspectives. Front Neuroend. 2014;35(3):320-30. https://doi.org/10.1016/j.yfrne.2014.05.004.

38. Donner NC, Lowry CA. Sex differences in anxiety and emotional behavior. Pflugers Arch Eur J Physiol. 2013;465(5):601-26.

39. Catuzzi JE, Beck KD. Anxiety vulnerability in women: A two-hit hypothesis Exp Neurol. 2014;259:75-80. https://doi.org/10.1016/j.expneurol.2014.01. 023.

40. Leach LS, Christensen H, Mackinnon AJ, Windsor TD, Butterworth P. Gender differences in depression and anxiety across the adult lifespan: The role of psychosocial mediators. Soc Psychiatry Psychiatr Epidemiol. 2008:43(12):983-98.

41. Findyartini A, Greviana N, Putera AM, Sutanto RL, Saki VY, Felaza E. The relationships between resilience and student personal factors in an undergraduate medical program. BMC Med Educ. 2021;21:113.

42. Peng L, Zhang J, Li M, Li P, Zhang Y, Zuo X, et al. Negative life events and mental health of Chinese medical students: the effect of resilience, personality and social support. Psychiatry Res. 2012;196:138-41. https:// doi.org/10.1016/j.psychres.2011.12.006.

43. Shi M, Liu L, Wang ZY, Wang L. The mediating role of resilience in the relationship between big five personality and anxiety among chinese medical students: a cross-sectional study. PLoS One. 2015;10(3):e0119916.

44. Cheng J, Zhao YY, Wang J, Sun YH. Academic burnout and depression of Chinese medical students in the pre-clinical years: the buffering hypothesis of resilience and social support. Psychol Health Med. 2019;25(9):1094. https://doi.org/10.1080/13548506.2019.1709651.

45. Tempski P, Santos IS, Mayer FB, Enns SC, Perotta B, Paro HBMS, et al. Relationship among medical student resilience, educational environment and quality of life. PLoS One. 2015;10(6):e0131535.

46. Shi $M$, Wang $X$, Bian $Y$, Wang $L$. The mediating role of resilience in the relationship between stress and life satisfaction among Chinese medical students: a cross-sectional study. BMC Med Educ. 2015;15:16

47. Schiller JH, Stansfield RB, Belmonte DC, Purkiss JA, Reddy RM, House JB, et al. Medical students' use of different coping strategies and relationship with academic performance in preclinical and clinical years. Teach Learn Med. 2018:30(1):15-21. https://doi.org/10.1080/10401334.2017.1347046.

48. Moffat KJ, McConnachie A, Ross S, Morrison JM. First year medical student stress and coping in a problem-based learning medical curriculum. Med Educ. 2004;38:482-91.

49. Farrell SM, Moir F, Molodynski A, Bhugra D. Psychological wellbeing, burnout and substance use amongst medical students in New Zealand. Int Rev Psychiatry. 2019;31 (7-8):630-6. https://doi.org/10.1080/09540261. 2019.1681204.

50. Merlo LJ, Curran JS, Watson R. Gender differences in substance use and psychiatric distress among medical students: A comprehensive statewide evaluation. Subst Abus. 2017;38(4):401-6.

51. Cheng C, Lau H-PB, Chan MPS. Coping flexibility and psychological adjustment to stressful life changes: a meta-analytic review. Psychol Bull. 2014;140(6):1582-607.

52. Freire C, Ferradás MDM, Núñez JC, Valle A. Coping flexibility and eudaimonic well-being in university students. Scand J Psychol. 2018;59(4):433-42.

53. Engel GL. The need for a new medical model: a challenge for biomedicine. Science (80- ). 1977:196(4286):129. 
54. Shankman SA, Lewinsohn PM, Klein DN, Small JW, Seeley JR, Altman SE. Subthreshold conditions as precursors for full syndrome disorders: a 15-year longitudinal study of multiple diagnostic classes. J Child Psychol Psychiatry. 2009;50(12):1485-94.

55. Scott J, Martin NG, Parker R, Couvy-Duchesne B, Medland SE, Hickie I. Prevalence of self-reported subthreshold phenotypes of major mental disorders and their association with functional impairment, treatment and full-threshold syndromes in a community-residing cohort of young adults. Early Interv Psychiatry. 2021:15(2):306-13.

56. Balázs J, Miklõsi M, Keresztény Á, Hoven CW, Carli V, Wasserman C, et al. Adolescent subthreshold-depression and anxiety: psychopathology, functional impairment and increased suicide risk. J Child Psychol Psychiatry. 2013;54(6):670-7.

57. Duffy A, Keown-Stoneman C, Goodday S, Horrocks J, Lowe M, King N, et al. Predictors of mental health and academic outcomes in first-year university students: Identifying prevention and early-intervention targets. BJPsych Open. 2020;6(3):e46.

58. Dyrbye LN, Sciolla AF, Dekhtyar M, Rajasekaran S, Allgood JA, Rea M, et al. Medical school strategies to address student well-being: a national survey. Acad Med. 2019;94(6):861-8.

59. Jacob R, Li T, Martin Z, Burren A, Watson P, Kant R, et al. Taking care of our future doctors: a service evaluation of a medical student mental health service. BMC Med Educ. 2020;20:172.

60. Dederichs M, Weber J, Muth T, Angerer P, Loerbroks A. Students' perspectives on interventions to reduce stress in medical school: A qualitative study. PLoS One. 2020;15(10):e0240587. https://doi.org/10.1371/journal. pone.0240587.

61. Brower KJ. Professional stigma of mental health issues: physicians are both the cause and solution. Acad Med. 2021;96(5):635-40.

62. Slavin SJ, Chibnall JT. Finding the why, changing the how: Improving the mental health of medical students, residents, and physicians. Acad Med. 2016;91(9):1194-6.

63. Slavin SJ. Medical student mental health: culture, environment, and the need for change. JAMA. 2016;316(21):2195-6.

64. Wasson LT, Cusmano A, Meli L, Louh I, Falzon L, Hampsey M, et al. Association between learning environment interventions and medical student well-being a systematic review. JAMA. 2016:316(21):2237-52.

65. Fried El. The 52 symptoms of major depression: Lack of content overlap among seven common depression scales. J Affect Disord. 2017;208:1917. https://doi.org/10.1016/j.jad.2016.10.019.

66. Thiemann P, Brimicombe J, Benson J, Quince T. When investigating depression and anxiety in undergraduate medical students timing of assessment is an important factor - A multicentre cross-sectional study. BMC Med Educ. 2020;20:125.

\section{Publisher's Note}

Springer Nature remains neutral with regard to jurisdictional claims in published maps and institutional affiliations.

Ready to submit your research? Choose BMC and benefit from:

- fast, convenient online submission

- thorough peer review by experienced researchers in your field

- rapid publication on acceptance

- support for research data, including large and complex data types

- gold Open Access which fosters wider collaboration and increased citations

- maximum visibility for your research: over $100 \mathrm{M}$ website views per year

At BMC, research is always in progress.

Learn more biomedcentral.com/submissions 